\title{
Case Report: "Spina Ventosa" Tuberculous Dactylitis in a 2 Year Old Boy - A Very Rare Disease
}

\author{
Ranadeb Bandyopadhyay ${ }^{*}, 1$, Arindam Mukherjee ${ }^{1}$ and Rajib Kumar Mondal ${ }^{2}$
}

Department of ${ }^{1}$ Orthopaedics, ${ }^{2}$ Departmentof Pathology, Bankura Sammilani Medical College, Gobindanagar, P.O. Kenduadihi, Bankura, Pin - 722102, India

\begin{abstract}
Tuberculous infection of metacarpals, metatarsals and phalanges is known as tuberculous dactylitis. There is a spindle shaped expansion of the short tubular bones due to tuberculous granuloma. Hence it is also known as spina ventosa. In our case, a two year old boy with a swelling in the metacarpal was provisionally diagnosed as enchondromata while the possibility of spina ventosa was kept in mind. He was posted for excision of the metacarpal followed by bone grafting. Histopathological examination report confirmed it as spina ventosa.
\end{abstract}

Keywords: Tuberculous dactylitis, tuberculous granuloma, spina ventosa, spindle shaped expansion.

\section{INTRODUCTION}

Tuberculous infection of metacarpals, metatarsals and phalanges of hands and feet is known as tuberculous dactylitis [1]. The hand is more frequently involved than the foot. Tuberculosis of the metacarpals, metatarsals and phalanges is uncommon after the age of 5years. In children the disease may occur in more than one short tubular bone at a time. There are not many reports devoted to tuberculous dactylitis.

\section{CASE REPORT}

A 2 year old boy was presented with a swelling of right middle metacarpal for last 4 months. The swelling was initially small gradually increasing in size. It was associated with dull aching pain for the last 3 months. There was no history of any trauma. However, h/o exposure to pulmonary tuberculosis was present.

On examination, a semi tubular swelling $5 \mathrm{~cm} * 2 \mathrm{~cm}$ was noted over right $3^{\text {rd }}$ metacarpal. The swelling was bony hard, fixed to underlying bone, tender and local temperature slightly raised.

A radiograph of the right hand showed a cystic expansile lesion of right $3^{\text {rd }}$ metacarpal. The margins were well defined with internal septations and associated cortical sclerosis. There was cortical destruction but no periosteal reaction.

A provisional diagnosis of enchondromata was made, while tuberculous dactylitis was kept in mind. Differential diagnoses were osteoblastoma, syphilis. The boy was recommended for surgery and excision of $3^{\text {rd }}$ metacarpal was done followed by strut graft from ipsilateral fibula. The specimen sent for HPE showed epitheloid granuloma suggestive of tuberculosis. Mantoux test was positive and sputum for AFB also revealed positive result (Figs. 1, 2).

\footnotetext{
*Address correspondence to this author at the Department of Orthopaedics, Bankura Sammilani Medical College, Gobindanagar, P.O. Kenduadihi, Bankura, Pin - 722102, India; Tel: +919434479225

E-mail: ranadeb.b@rediffmail.com
}

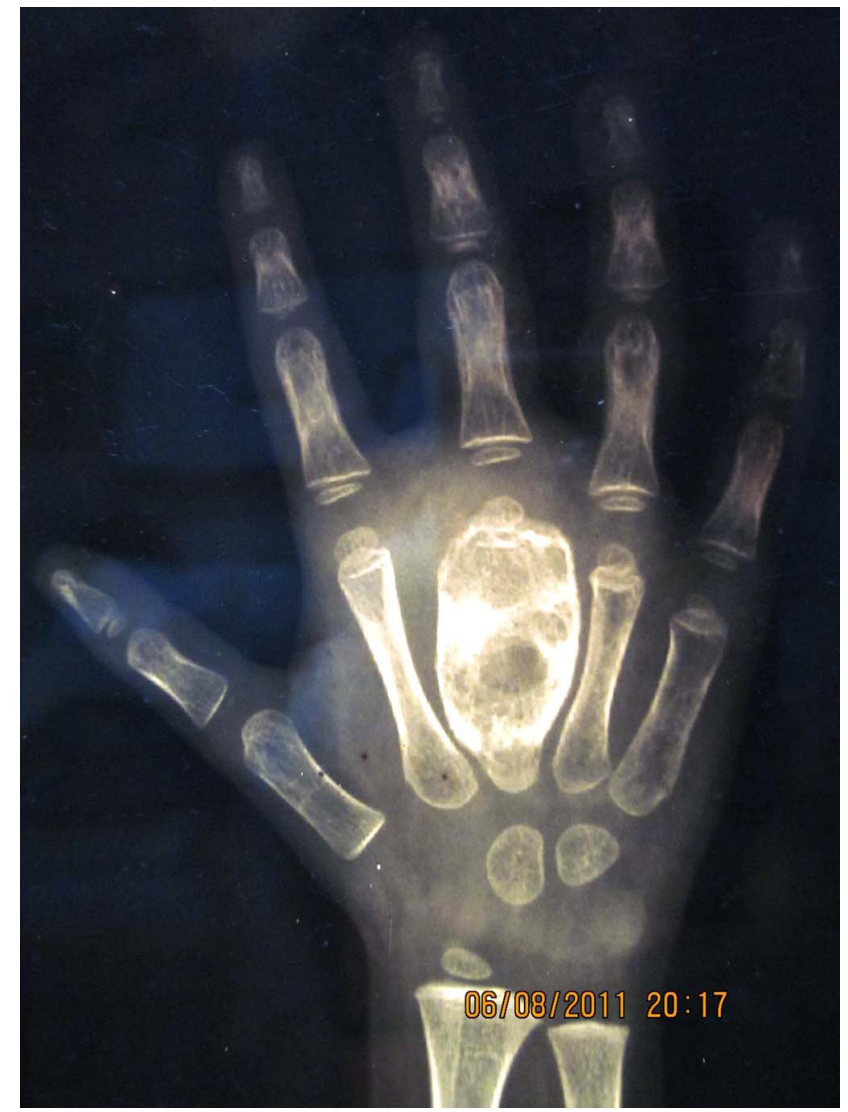

Fig. (1). X-ray right hand A-P pre operative showing osteolytic lesion in $3^{\text {rd }}$ metacarpal.

\section{DISCUSSION}

Bone and joint tuberculosis occur in 1-5\% children who have untreated initial pulmonary tuberculosis and spread to the skeletal system during the initial infection via the lymphohaematogenous route [2]. The skeletal infection becomes symptomatic within 1-3 years after the initial infection. $85 \%$ of children with tuberculous dactylitis are younger than 6years of age and its incidence among children 
with tuberculosis was reported to be $0.65 \%-6.9 \%(3)$. The bones of hands are more frequently affected than the bones of feet with the proximal phalanx of index and middle finger more frequently affected.

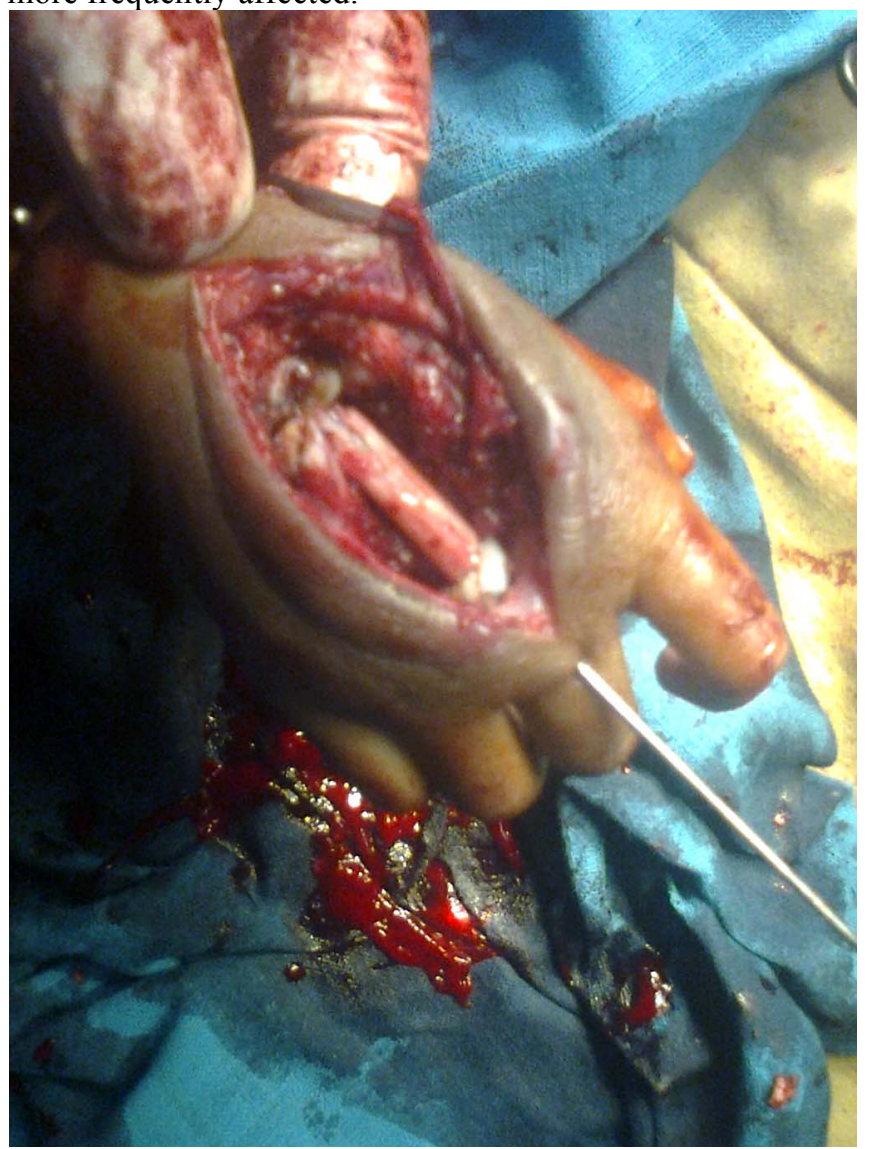

Fig. (2). Excision of the lesion and fibular grafting.

The condition usually presents as a painless swelling of a digit of a few months duration. The radiographic feature of cystic expansion of short tubular bones has led to the name of SPINA VENTOSA being given to tuberculosis dactylitis of the short bones of the hand $[3,4]$. Periosteal reaction and sequestra are not common but may occur. Sclerosis may be seen in long standing cases.

During childhood, these short tubular bones have a lavish blood supply through a large nutrient artery entering almost in the middle of the bone. The first inoculum of infection is lodged in the centre of the marrow cavity and the interior of the short tubular bone is converted gradually into a tuberculosis granuloma. This leads to a spindle shaped expansion of the bone (SPINA VENTOSA) with the occlusion of the nutrient artery of the involved bone and the destruction of internal lamellae (or formation of sequestra). In natural course, the disease heals with shortening of the involved bone and deformity of the neighbouring joint.

Tuberculous dactylitis needs to be differentiated on one hand from chronic pyogenic osteomyelitis and syphilitic dactylitis and on the other hand from neoplastic conditions with lytic lesions (enchondromata and fibrous defect). Other rare granulomatous conditions which mimic tuberculous infection are mycotic infection, sarcoidosis and brucellosis.

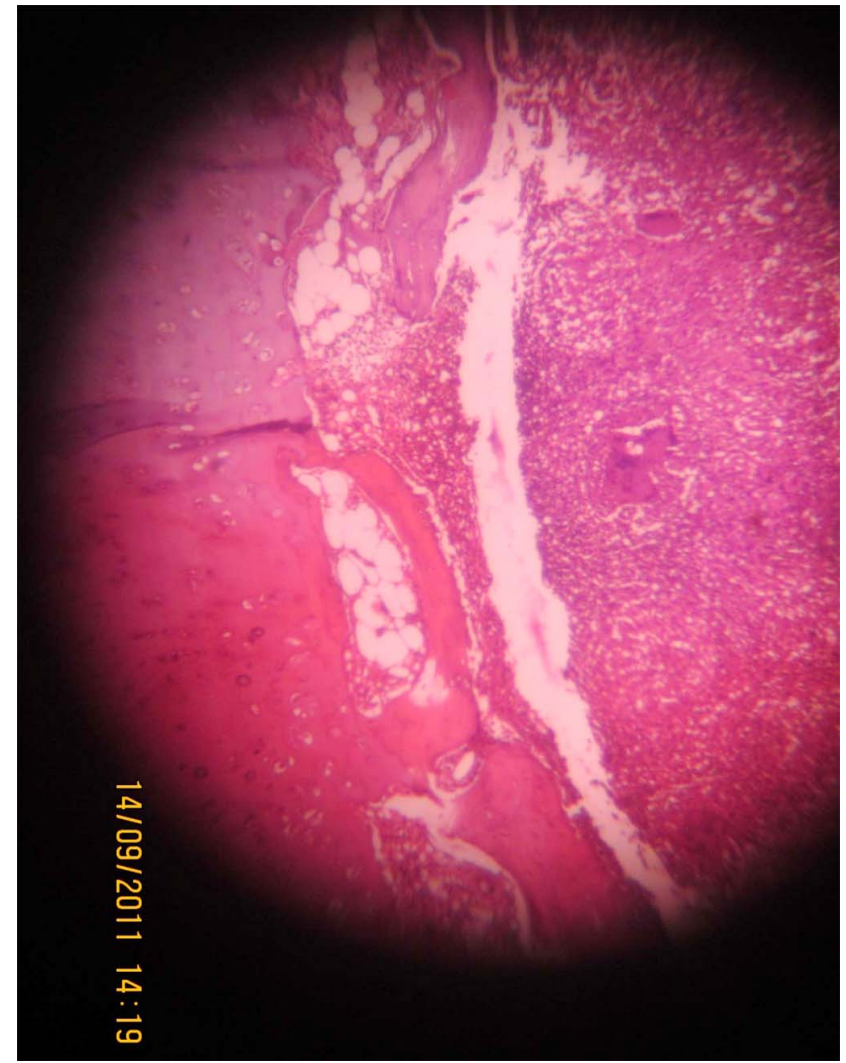

Fig. (3). Photomicrograph showing epitheloid granuloma with bony trabeculae (Haematoxylin \& eosin, ${ }^{* 40}$ ).

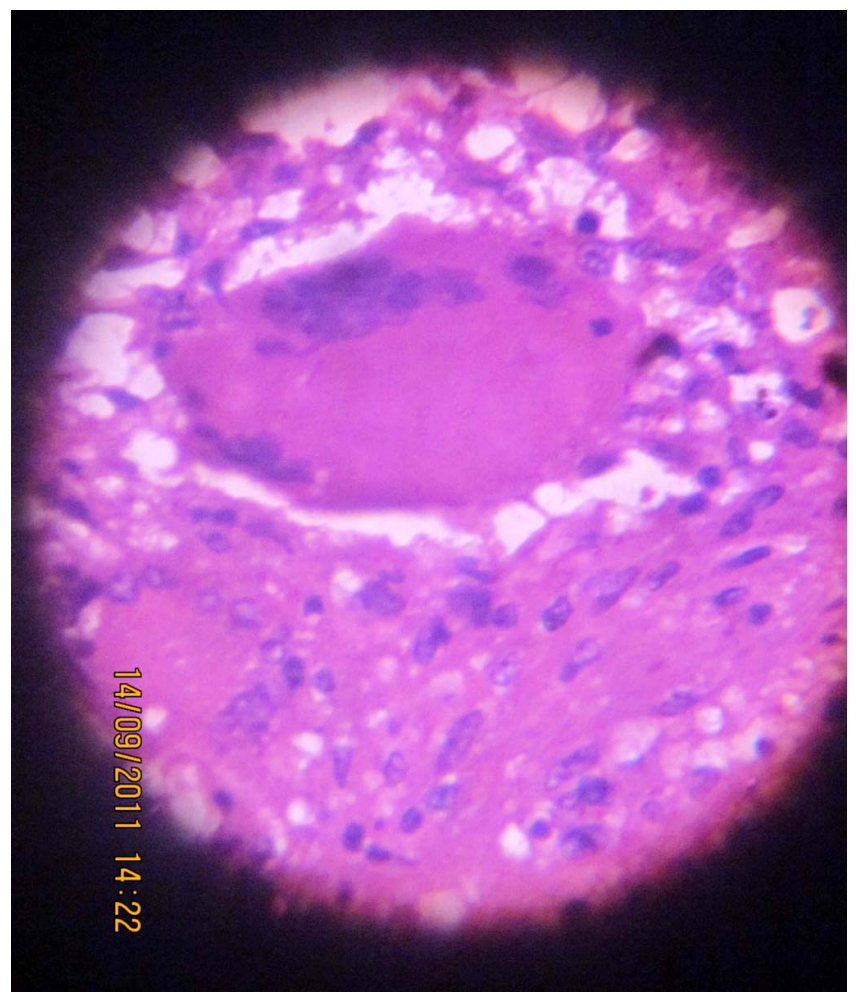

Fig. (4). Photomicrograph showing epitheloid granuloma in high power (Haematoxylin \& eosin,*400). 
Management is essentially done by anti tubercular drugs, rest to the part in functioning position and early active exercise of the involved parts or joints. In patients with unfavorable response or recurrence of infection, surgical debridement is justified. If a metacarpo-phalangeal, metatarso-phalangeal or interphalangeal joint is ankylosed in awkward position excision arthroplasty or corrective osteotomy is indicated. If a finger has ankylosed of more than one joint, is grossly deformed scarred and interfering with normal functioning it may be wise to ampute the finger or the corresponding ray $[5,6]$.

In our case a provisional diagnosis of enchondromata was made, while tuberculous dactylitis was kept in mind. Differential diagnoses were osteoblastoma, syphilis. Surgery was conducted and excision of $3^{\text {rd }}$ metacarpal was done followed by strut graft from ipsilateral fibula done. The specimen sent for HPE showed epitheloid granuloma suggestive of tuberculosis. Mantoux test was positive and sputum for AFB also revealed positive result. ATD was started post-operatively (Figs. 3, 4).

\section{CONCLUSION}

In general tuberculous dactylitis is treated with ATDs. However, in this case, pre-operative diagnosis could not be made. Therefore, we started with surgical intervention and anti-tubercular drugs were added post-operatively. After completion of ATD therapy, at one year follow-up, patient was completely disease free and healthy.

\section{ACKNOWLEDGEMENT}

Declared none.

\section{CONFLICT OF INTEREST}

Declared none.

\section{REFERENCES}

[1] Tuli SM. Tuberculosis of short tubular bones. Tuberculosis of the skeletal system. New Delhi: Jaypee Brothers Medical Publishers 1991; pp. 164-5.

[2] Kushwaha RA, Kant S, Verma SK, Sanjay, Mehra S. Case report: Isolated metacarpal bone tuberculosis-A case report. Lung India 2008; 25(1): 17-9.

[3] Gyanshankar PM, Dhamgaye TM, Amol BF. Spina ventosa discharging tubercle bacilli--a case report. Indian J Tuberc 2009; 56(2):100-3.

[4] Malik S, Joshi S, Tank JS. Cystic bone tuberculosis in children--a case series. Indian J Tuberc 2009; 56(4): 220-4.

[5] Roy AK, Khanduri S, Girisha KM. Spot the diagnosis: Fusiform swellings of fingers in a 3-year-old girl. J Postgrad Med 2006; 52(4): 314

[6] Salimpour R, Salimpour P. Picture of the month, Tuberculous dactylitis. Arch Pediatric Adolescs Med 1997; 151(8): 851-2. 